\title{
Collective Modes and Structural Modulation in Ni-Mn-Ga(Co) Martensite Thin Films Probed by Femtosecond Spectroscopy and Scanning Tunneling Microscopy
}

\author{
M. Schubert, ${ }^{1}$ H. Schaefer, ${ }^{1,2}$ J. Mayer, ${ }^{1}$ A. Laptev, ${ }^{1}$ M. Hettich, ${ }^{1}$ M. Merklein, ${ }^{1}$ C. He, ${ }^{1}$ C. Rummel, ${ }^{1}$ O. Ristow, \\ M. Großmann, ${ }^{1}$ Y. Luo, ${ }^{3}$ V. Gusev, ${ }^{1,4}$ K. Samwer, ${ }^{3}$ M. Fonin, ${ }^{1}$ T. Dekorsy, ${ }^{1}$ and J. Demsar ${ }^{1,2,5}$ \\ ${ }^{1}$ Department of Physics, University of Konstanz, 78457 Konstanz, Germany \\ ${ }^{2}$ Institute of Physics, Ilmenau University of Technology, 98693 Ilmenau, Germany \\ ${ }^{3}$ I. Physikalisches Institut, Georg-August-Universität Göttingen, 37073 Göttingen, Germany \\ ${ }^{4}$ Laboratoire d'Acoustique, LAUM, UMR CNRS 6613, LUNAM Université, Université du Maine, 72085 Le Mans, France \\ ${ }^{5}$ Institute of Physics, Johannes Gutenberg-University Mainz, 55128 Mainz, Germany
}

(Received 19 January 2015; published 13 August 2015)

\begin{abstract}
The origin of the martensitic transition in the magnetic shape memory alloy Ni-Mn-Ga has been widely discussed. While several studies suggest it is electronically driven, the adaptive martensite model reproduced the peculiar nonharmonic lattice modulation. We used femtosecond spectroscopy to probe the temperature and doping dependence of collective modes, and scanning tunneling microscopy revealed the corresponding static modulations. We show that the martensitic phase can be described by a complex charge-density wave tuned by magnetic ordering and strong electron-lattice coupling.
\end{abstract}

PACS numbers: 71.45.Lr, 78.47.-p, 81.30.Kf, 81.70.Fy

Magnetic shape memory alloys present a new type of multifunctional materials, which display strong coupling between the magnetic and structural degrees of freedom. The ferromagnetic Ni-Mn-Ga alloy serves as a prototype system, displaying a giant $12 \%$ magnetic-field-induced strain in its low-temperature $(T)$ martensitic phase $(M$ phase) [1,2]. Since in Ni-Mn-Ga the $M$-phase transition can be tuned far above the room temperature by changing stoichiometry or doping [3,4], this alloy is of special technological interest $[1,2,5]$.

The rich phase diagram of $\mathrm{Ni}_{2+x+y} \mathrm{Mn}_{1-x} \mathrm{Ga}_{1-y}$ is characterized by a complex sequence of phase transitions. In its high- $T$ (austenite) phase, Ni-Mn-Ga has a cubic $L 2_{1}$ Heusler structure. At the $M$-phase transition temperature $T_{M}$, the lattice undergoes a transformation, which can be described by a periodic shuffling of (011) planes along the [011] direction [6]. Depending on the stoichiometry and the residual stress, the resulting low- $T M$ phase is commonly found to have either tetragonal or orthorhombic symmetry, with a modulation period of ten $(10 M)$ or $14(14 M)$ atomic layers [6,7], respectively. For specific stoichiometries, e.g., $\mathrm{Ni}_{2+x} \mathrm{Mn}_{1-x}$ Ga with $x \lesssim 0.1$, a premartensitic phase (PM phase) is observed above $T_{M}$ [3] with the transition temperature $T_{\mathrm{PM}}$ up to $50 \mathrm{~K}$ above $T_{M}[3,8]$. In the PM phase, the lattice displays a harmonic threefold modulation with the wave vector $\mathbf{q}_{\mathrm{PM}}=q_{\max }\left(\frac{1}{3}, \frac{1}{3}, 0\right)$.

Inelastic neutron scattering studies of the high- $T$ cubic phase [9] showed a dramatic softening of the TA-2 phonon branch at $\mathbf{q}_{\mathrm{PM}}$, indicative of a Kohn anomaly. These observations [9-12], together with the observed opening of a pseudogap at $T_{\mathrm{PM}}[8,13]$, suggest an electronic instability within the Peierls scenario to be driving the PM-phase transition. The observation of a phase mode in the $10 M$ phase [14] also suggested the electronic instability. In fact, the electronic band structure studies $[11,15,16]$ revealed the possible Fermi surface nesting conditions for all of the observed structural modulations. However, for the case of the $14 M$ martensite, it was argued that the modulated phase can be constructed from nonmodulated martensitic unit cells $[17,18]$. This adaptive martensite scenario [17-19] recently received considerable attention.

To determine which theory gives a more appropriate physical picture of the phase transition in Ni-Mn-Ga, we applied femtosecond time-resolved spectroscopy combined with scanning tunneling microscopy (STM) on a series of undoped and Co-doped Ni-Mn-Ga films. Alloying with cobalt results in a shift of $T_{M}$ far above room temperature and is thus of vital importance for technological applications [20]. While STM provides direct access to static structural modulation, the bulk sensitive femtosecond optical spectroscopy is particularly useful for studying low-energy collective excitations, like Raman active phonons [21] or amplitude modes of charge-density wave (CDW) systems [22-25].

In this Letter, we demonstrate the existence of collective modes in the $14 M$ phase that stem from the coupling of the lattice to the underlying electronic instability. We show that the stacking sequence recorded by STM, which is close to the structure proposed by the adaptive martensite scenario $[17,18]$, can be consistently explained by the higher-order coupling between the electronic order and the lattice. Strong support for this scenario is provided by the data obtained on films lightly doped with cobalt. In Ni-Mn-Ga(Co), the STM data reveal a change in the modulation periodicity, which manifests itself by a dramatic change in the collective mode's eigenfrequencies. This indicates the 
importance of magnetic ordering on the electron-lattice coupling and the resulting structural transformation.

$\mathrm{Ni}-\mathrm{Mn}-\mathrm{Ga}$ thin films of various compositions were investigated [26]. All samples were characterized by XRD, EDX, and SQUID measurements. Additionally, some samples were investigated by STM. In order to reach atomic resolution, the surfaces of the samples were treated in ultrahigh vacuum (labeled with the letter " $S$ " [26]).

Time-resolved reflectivity dynamics at $800 \mathrm{~nm}$ were recorded in two configurations [26], providing access both to the $q=0$ modes in the terahertz range as well as to long wavelength acoustic modes via acoustic echoes [21,29]. Figure 1 shows the $T$ dependence of the transient reflectivity over the first $10 \mathrm{ps}$ recorded on sample $B 2$ $\left(\mathrm{Ni}_{51.0} \mathrm{Mn}_{23.4} \mathrm{Ga}_{25.6}\right)$ and the Co-doped sample $C 1$ $\left(\mathrm{Ni}_{47.9} \mathrm{Mn}_{25.5} \mathrm{Ga}_{23.6} \mathrm{Co}_{3.0}\right)$. In both samples, no highfrequency modes are observed in the austenite phase, which is consistent with the absence of optical phonons below $\approx 4 \mathrm{THz}$ [9].

In sample $B 2$, the high-frequency oscillations can be observed in both the $M$ phase and the PM phase. A fast Fourier transform (FFT) of the low- $T$ data in Fig. 1(c) reveals a strong mode at $\approx 1.4 \mathrm{THz}$ and a distinct shoulder towards lower frequencies. In Co-doped samples [Fig. 1(b)], the $M$ phase is also characterized by the oscillatory response, however with dramatically different mode frequencies. As elaborated below, these $q=0$ modes are coherently excited collective modes (amplitudons) of the system $[22,23]$.

Figure 2 shows the $T$ dependence of the FFT spectra for samples $B 2$ and $C 1$, together with the extracted mode frequencies for several undoped and Co-doped samples. In (a)

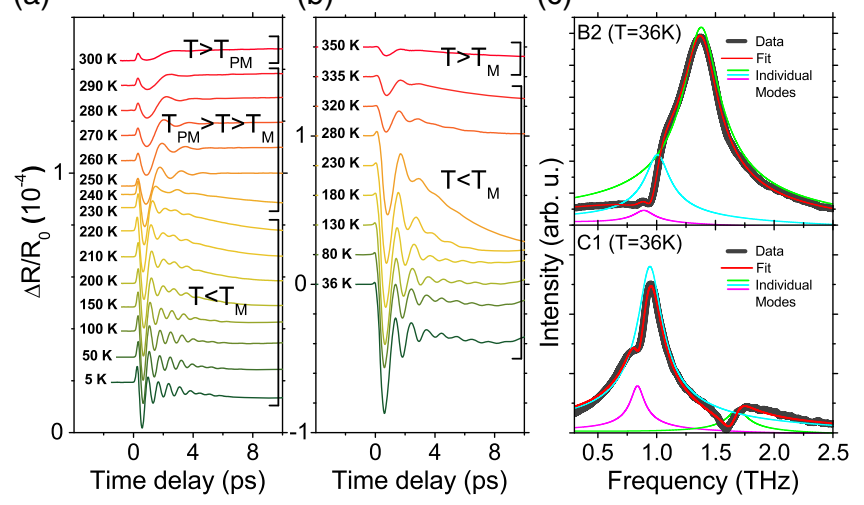

FIG. 1 (color online). The photoinduced reflectivity traces recorded on (a) an undoped sample $B 2$ and (b) a Co-doped sample $C 1$ at different temperatures. The different phases are indicated by large brackets. (c) The FFT spectra of the oscillatory response for the two samples in the $M$ phase. The spectra are fitted as a sum of Lorentzian modes, represented by differently colored lines. The overall fit is shown as a red line on top of the measured data in dark gray.

the PM phase (observed only in near stoichiometric $B 2$ and $B 3$ ), the mode at $\approx 0.8 \mathrm{THz}$ is observed above $T_{M}$, displaying strong softening upon approaching $T_{\mathrm{PM}}$. This mode has also been observed in the PM phase of bulk Ni-Mn-Ga samples [30] and was identified as an amplitude mode. Indeed, this assignment is consistent with the proposed Fermi-surface nesting scenario [8-11,13].

The origin of the modes in the $M$ phase is not so obvious. In undoped thin films, the main mode is centered at $\approx 1.4 \mathrm{THz}$, while a much weaker mode is observed at $\approx 1 \mathrm{THz}$ [Fig. 1(c)]. In stress-free bulk samples [30], as well as in low-stress, thick films (B3), only the $1.4 \mathrm{THz}$ mode can be clearly resolved. As $T$ is raised towards $T_{M}$, we observe a large decrease of the mode's frequencies by $\approx 20 \%$, until they disappear near $T_{M}$. In Co-doped films, the behavior is qualitatively similar (see Figs. 1 and 2), but the mode's frequencies are severely altered. The frequency of the dominant mode is about $40 \%$ lower, centered at $\approx 1 \mathrm{THz}$. In addition, there is a pronounced mode at $\approx 1.7 \mathrm{THz}$ and a weaker one at $\approx 0.9 \mathrm{THz}$.

The system shows a strong mode softening and strong changes of the mode frequencies upon weak doping with Co. This is expected if the phase transition is driven by the electronic instability such as in the CDW scenario [22] as opposed to by simple zone folding (adaptive martensite scenario). Indeed, in standard CDW systems [22,24,25] numerous Raman active $(q=0)$ modes appear in the CDW phase, showing comparable softening upon approaching the CDW ordering temperature $T_{\mathrm{CDW}}$.

In a typical (one-dimensional) Peierls-like CDW system, a phase transition is characterized by the appearance of a sinusoidal modulation of the conduction electron density and the corresponding lattice displacements with $\mathbf{q}_{\mathrm{CDW}}=2 \mathbf{k}_{F}$. It was shown recently $[22,25]$ that the appearance of $q=0$ collective modes below $T_{\mathrm{CDW}}$ can

(a)
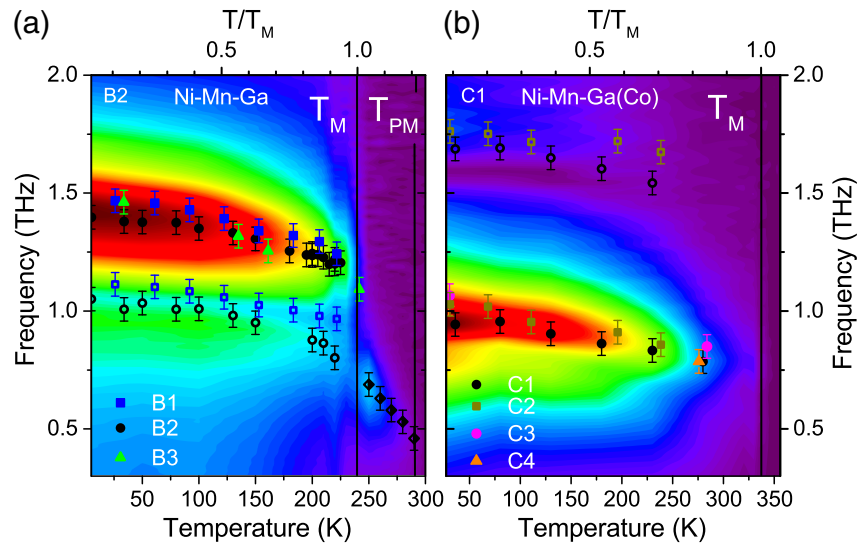

FIG. 2 (color online). The $T$ dependence of the FFT spectra for samples (a) $B 2$ and (b) $C 1$. The frequencies of dominant modessee also Fig. 1(c)—are shown by black symbols. The mode frequencies recorded on other samples [26] are also plotted with respect to the reduced temperature $\left(T / T_{M}\right)$. 
be well explained in terms of a linear coupling between the electronic part of the order parameter and $\mathbf{q}_{\mathrm{CDw}}$ phonons of the high- $T$ phase. In the simplest case of an incommensurate CDW with inversion symmetry, the electronic part of the order parameter may be represented by a complex $\Delta$, whereby $\boldsymbol{\Delta}\left(-\mathbf{q}_{\mathrm{CDW}}\right)=\boldsymbol{\Delta}^{*}\left(\mathbf{q}_{\mathrm{CDW}}\right)$. The linear coupling of $\boldsymbol{\Delta}$ with normal state phonons at $\mathbf{q}_{\mathrm{CDW}}$ gives rise to a $\sum_{k} c_{k} \boldsymbol{\Delta} \xi_{k}$ contribution to the free energy, where $\boldsymbol{\xi}_{k}\left(\mathbf{q}_{\mathrm{CDW}}\right)$ are normal coordinates of phonon modes having the same symmetry as $\boldsymbol{\Delta}$. Thus, each of these $\mathbf{q}_{\mathrm{CDW}}$ phonons is expected to result in one Raman active mode below $T_{\mathrm{CDW}}$. In the case of strong coupling, however, higher-order coupling terms, such as $\boldsymbol{\Delta}^{2}\left(\mathbf{q}_{\mathrm{CDW}}\right) \boldsymbol{\xi}_{k}\left(2 \mathbf{q}_{\mathrm{CDW}}\right)$, may need to be included. In this case, coupling with phonons at $2 \mathbf{q}_{\mathrm{CDW}}, 3 \mathbf{q}_{\mathrm{CDW}}, 4 \mathbf{q}_{\mathrm{CDW}}$, etc., would result in additional, though weaker, $q=0$ modes in the CDW phase $[22,25]$. The fundamental difference between the CDW scenario and the rigid zone folding is that these low- $T$ modes are collective modes of the system whose frequencies are strongly renormalized due to the coupling to the electronic order. This is also the reason for mode softening upon approaching $T_{\mathrm{CDW}}$.

To show that a CDW scenario can account for our observations, we performed STM studies on selected samples to investigate the static modulation of the surface. Figure 3(a) shows the schematic structure of an ideal $14 M$ martensite. The $5 \overline{2} 5 \overline{2}$ stacking along the [011] direction is reflected in an equivalent modulation of the surface along the [010] direction. Figure 3(b) shows the (001) surface of undoped sample $B 2 S$ in the $M$ phase. The atomic scale image, shown in Fig. 3(c), reveals a topography which is consistent with the idealized $5 \overline{2}$ stacking sequence with a periodicity of $14\{110\}$ atomic planes $(14 M)$. Larger area
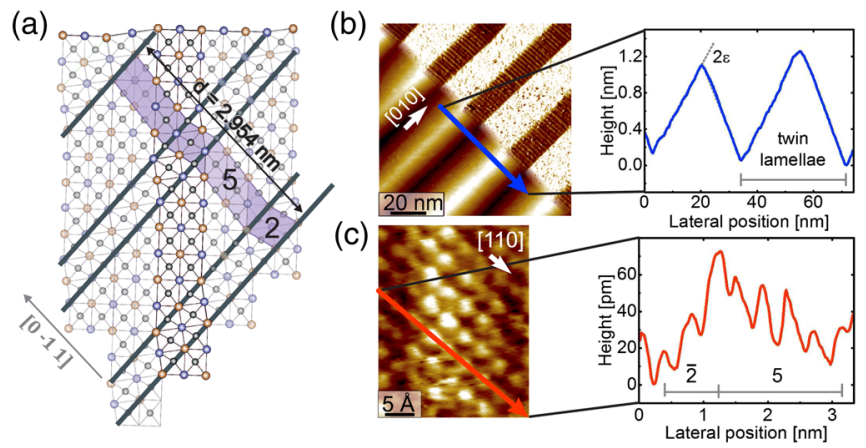

FIG. 3 (color online). (a) Schematic drawing of the (100) plane of an idealized $14 \mathrm{M}$ martensite. The nanotwin boundaries are denoted by solid green lines. (b) An STM image (sample $B 2 S$ ) of the atomically flat (001) surface, showing several twin lamellae (lower left, topography; upper right, topography gradient along the [110] direction). One of the two twin variant orientations reveals an additional out of plane corrugation. A height profile along the [100] direction is shown. The measured angle $\epsilon=$ $3.6 \pm 0.2^{\circ}$ is characteristic for the $14 M M$ phase. (c) Atomicresolution STM image of the out of plane corrugation showing a fourteen-layered superstructure with a $5 \overline{2}$ stacking sequence. scans, however, show a distribution of modulation variations. This leads to a larger number of $4 \overline{3}$, as well as $4 \overline{4}$ and $5 \overline{3}$ combinations. The average pair length was $7.2 \pm 0.1$ layers for sample $B 2 S$.

Clearly, a single harmonic modulation is insufficient to describe a stackinglike modulation of the $14 M$ phase. The FFT of the static atomic displacements of the idealized $5 \overline{2} 5 \overline{2}$ stacking structure along the [010] direction reveals that at least three modulation wave vectors are required to reproduce the structure, corresponding to $\mathbf{q}_{1}^{14 M}=$ $q_{\max }\left(\frac{4}{7}, \frac{4}{7}, 0\right), \mathbf{q}_{2}^{14 M}=q_{\max }\left(\frac{8}{7}, \frac{8}{7}, 0\right)=2 \mathbf{q}_{1}^{14 M}$, and $\mathbf{q}_{3}^{14 M}=$ $q_{\max }\left(\frac{12}{7}, \frac{12}{7}, 0\right)=3 \mathbf{q}_{1}^{14 M}$. These three wave vectors can indeed be resolved in our STM data on sample $B 2 S$ as shown in Fig. 4(a). The STM data recorded on Co-doped films show, however, somewhat different periodicity. The modulation period recorded on sample $C 1 S$ is about $13 \%$ larger, consistent with the modulation wave vector $q_{\max }\left(\frac{1}{2}, \frac{1}{2}, 0\right)$. Again, a component at twice the wave vector is clearly resolved, implying a nonharmonic modulation. The data suggest a commensurate modulation with $5 \overline{3}$ stacking and a periodicity of eight atomic layers (or four unit cells). We refer to an ideal $5 \overline{3}$ modulated structure as $8 M$. While this is not a commonly encountered phase in the Ni-Mn-Ga system, it has been reported in Mn-rich films [31].

In order to link the observed low- $T$ collective modes to specific phonons of the austenite phase, we analyze the published neutron scattering data $[9,10]$. The phonon dispersions along the modulation direction, $\mathbf{q}=q_{\max }(\xi, \xi, 0)$, recorded on a stoichiometric $\mathrm{Ni}_{2} \mathrm{MnGa}$ [9] are reproduced in Fig. 4(b). The pronounced dip in the dispersion near $\mathbf{q}_{\mathrm{CDW}}^{\mathrm{PM}}=q_{\max }\left(\frac{1}{3}, \frac{1}{3}, 0\right)$, which is present far above $T_{\mathrm{PM}}$, represents the Kohn anomaly leading to a harmonic threefold modulation in the PM phase. Indeed, the frequency of the collective mode just above $T_{M}$ - see Fig. 2(a) - can be well linked to the bare phonon frequency of a TA-2 mode at $\mathbf{q}_{\mathrm{CDW}}^{\mathrm{PM}}$ for $T \gg T_{\mathrm{PM}}$, supporting the
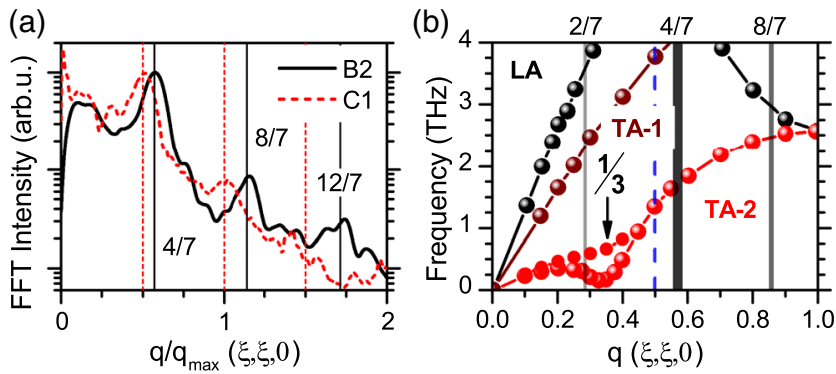

FIG. 4 (color online). (a) The FFT of the height profiles in the $M$ phase of samples $B 2 S$ and $C 1 S$. The periodicity in Co-doped sample $C 1 S$ is consistent with the dominant $5 \overline{3}$ stacking. (b) The low-energy phonon dispersion along the $(\xi, \xi, 0)$ direction just above $T_{\mathrm{PM}}$ (adapted from Ref. [9]). The high-temperature data (400 K) for the TA-2 branch are given by solid red circles. The vertical lines denote the wave vectors related to the $14 M$ (solid gray line) and $8 M$ (blue dashed line) modulated structures. 
assignment of this mode to the amplitude mode of the PM phase.

In the $M$ phase, the frequencies of dominant modes are distinctly different between the undoped and Co-doped samples. Within the CDW scenario, the frequencies of dominant modes at $T \ll T_{M}$ should be close to the bare phonon frequencies at $\mathbf{q}_{\mathrm{CDW}}$, yet renormalized due to coupling to the electronic order. Indeed, for undoped samples the frequency of the dominant mode $(\approx 1.4 \mathrm{THz})$ can be well linked to the frequency of the bare TA-2 mode at $\mathbf{q}_{1}^{14 M}$. The reduction of frequency of the main mode to $\approx 1 \mathrm{THz}$ in Co-doped samples can be attributed to the change in the CDW modulation wave vectors from $\mathbf{q}_{1}^{14 M}=q_{\max }\left(\frac{4}{7}, \frac{4}{7}, 0\right)$ to $\mathbf{q}_{1}^{8 M}=q_{\max }\left(\frac{1}{2}, \frac{1}{2}, 0\right)$, in accordance with the STM data.

As shown in Figs. 1 and 2, there are additional weaker modes in both undoped and Co-doped films. The weak mode at $\approx 1 \mathrm{THz}$ observed in thin undoped samples matches the dominant mode in Co-doped $(8 M)$ films. In single crystalline samples [30], as well as in thick films (B3), this mode is absent. Since in the nonstoichiometric thin films the STM data do show a high number of $5 \overline{3}$ stacking sequences, we attribute this mode to the mixing of the $8 M$ phase into the dominant $14 M$ phase. This can be caused both by nonstoichiometry and by the strain induced by the film-substrate lattice mismatch.

The nonharmonic static modulation suggests that higherorder coupling terms may be expected. When backfolded into the first Brillouin zone of the high- $T$ phase, the second and the third wave vectors of the $14 M$ structure are given by $\mathbf{q}_{2}^{14 M}=q_{\max }\left(\frac{6}{7}, \frac{6}{7}, 0\right)$ and $\mathbf{q}_{3}^{14 M}=q_{\max }\left(\frac{2}{7}, \frac{2}{7}, 0\right)$, respectively. Since these phonons are optical in nature, the corresponding low- $T$ amplitude modes should appear at frequencies above $4 \mathrm{THz}$ [9]. Given the higher-order coupling, implying lower amplitudes [25], as well as the reduced sensitivity of the technique at high frequencies, their observation is beyond our reach.

In the Co-doped $(8 M)$ samples, $\mathbf{q}_{2}^{8 M}=q_{\max }(1,1,0)$ corresponds to the zone edge phonon, while $\mathbf{q}_{3}^{8 M}$ equals $\mathbf{q}_{1}^{8 M}$. Here the mode at $\approx 1.75 \mathrm{THz}$ could originate from quadratic coupling of the electronic part of the order parameter to the zone-edge phonon. Alternatively, this mode could results also from a coupling of the electronic order to a pair of acoustic phonons, whose sum of wave vectors matches $\mathbf{q}_{1}^{8 M}$. Such invariants are also allowed in commensurate systems and should be more pronounced in the case of quadrupling of the unit cell $(8 M)$ as in the $14 M$ phase.

Finally, the origin of the change in periodicity upon doping with $2 \%-4 \%$ of Co should be addressed. The CDW scenario suggests the change is a result of a change in the nesting wave vector. While the data on the evolution of the Fermi surface with doping are unavailable, there are numerous observations that support such a scenario. First of all, calculations of the static electronic susceptibility [15] suggest the contributions from the majority and minority spin are considerably different [16]. Since doping with Co is found to strongly affect both the total magnetic moment [32-34] as well as densities of states in the minority- and majority-spin channels $[20,32]$, it is likely that the peak in the static electronic susceptibility is pushed from $q_{\max }\left(\frac{4}{7}, \frac{4}{7}, 0\right)$ towards the next major commensurability wave vector $q_{\max }\left(\frac{1}{2}, \frac{1}{2}, 0\right)$. The impact of magnetism on the Fermi surface topology and the formation of modulated structures was studied also by $a b$ initio methods $[35,36]$. These also suggest the phase transitions in Ni-Mn-Ga result from an interplay between vibrational and magnetic effects.

In summary, we present a femtosecond optical study of undoped and Co-doped Ni-Mn-Ga thin films combined with STM measurements. Time-resolved data reveal the appearance of a number of low-frequency collective modes in the $M$ phase. All modes display a pronounced softening upon approaching the $M$-phase transition from below, similar to amplitude modes of CDW systems. The assignment is consistent with the recent structural study on the photoinduced quenching and recovery of the martensitic modulation in off-stoichiometric Ni-Mn-Ga film [37]. Doping with $\approx 3 \%$ of Co results in dramatic changes in mode frequencies, while the STM data reveal a change in the modulation periodicity. When comparing the mode frequencies to the known phonon dispersion in the stoichiometric $\mathrm{Ni}_{2} \mathrm{MnGa}$, we conclude that these collective modes are a result of strong coupling of the lattice to the underlying electronic instability. Our results imply a complex CDW ground state, with the nonharmonic martensitic modulation being a result of the higher-order coupling to the lattice. The nonharmonic lattice modulation can be consistently explained within the electronic instability scenario and higher-order coupling. Our results demonstrate strong dependence of the structural modulation to the underlying, spin-dependent, band structure. The interplay between the magnetic ordering and strong electron-lattice coupling can well account for the large variety of modulations found in the Ni-Mn-Ga system, since small changes in the two, due to stress or composition changes, can easily lead to changes in structural modulation.

The authors acknowledge financial support by the DFG (SFB 767) and the BMBF (MSM-Sens, Projects No. 13N10061 and No. 13N10062) and the Carl Zeiss Foundation (H. S.). We thank A. Pashkin, M. Körber, and M. Altenburg for supporting the study and A. Bruchhausen, V. V. Kabanov, and H. J. Elmers for helpful discussions.

M. S. and H. S. contributed equally to this work.

[1] A. Sozinov, A. A. Likhachev, N. Lanska, and K. Ullakko, Appl. Phys. Lett. 80, 1746 (2002).

[2] M. Chmielus, X. X. Zhang, C. Witherspoon, D. C. Dunand, and P. Mullner, Nat. Mater. 8, 863 (2009). 
[3] P. Entel, V. D. Buchelnikov, V. V. Khovailo, A. T. Zayak, A. W. Adeagbo, M.E. Gruner, H. C. Herper, and E. F. Wassermann, J. Phys. D 39, 865 (2006).

[4] I. Takeuchi et al., Nat. Mater. 2, 180 (2003).

[5] E. Pagounis, A. Laptev, J. Jungwirth, M. Laufenberg, and M. Fonin, Scr. Mater. 88, 17 (2014).

[6] V. V. Martynov and V. Kokorin, J. Phys. III (France) 2, 739 (1992).

[7] J. Pons, V. A. Chernenko, R. Santamarta, and E. Cesari, Acta Mater. 48, 3027 (2000).

[8] C. P. Opeil et al., Phys. Rev. Lett. 100, 165703 (2008).

[9] A. Zheludev, S. M. Shapiro, P. Wochner, and L. E. Tanner, Phys. Rev. B 54, 15045 (1996).

[10] L. Mañosa, A. Gonzàlez-Comas, E. Obrado, A. Planes, V. A. Chernenko, V. V. Kokorin, and E. Cesari, Phys. Rev. B 55, 11068 (1997).

[11] C. Bungaro, K. M. Rabe, and A. Dal Corso, Phys. Rev. B 68, 134104 (2003).

[12] A. Planes, E. Obradó, A. Gonzàlez-Comas, and L. Mañosa, Phys. Rev. Lett. 79, 3926 (1997).

[13] S. W. D’Souza, A. Rai, J. Nayak, M. Maniraj, R. S. Dhaka, S. R. Barman, D. L. Schlagel, T. A. Lograsso, and A. Chakrabarti, Phys. Rev. B 85, 085123 (2012).

[14] S. M. Shapiro, P. Vorderwisch, K. Habicht, K. Hradil, and H. Schneider, Europhys. Lett. 77, 56004 (2007).

[15] O. Velikokhatnyi and I. Naumov, Phys. Solid State 41, 617 (1999).

[16] T. D. Haynes, R. J. Watts, J. Laverock, Z. S. Mayor, M. A. Alam, J. W. Taylor, J. A. Duffy, and S. B. Dugdale, New J. Phys. 14, 035020 (2012).

[17] S. Kaufmann, U. K. Rössler, O. Heczko, M. Wuttig, J. Buschbeck, L. Schultz, and S. Fähler, Phys. Rev. Lett. 104, 145702 (2010).

[18] S. Kaufmann, R. Niemann, T. Thersleff, U. K. Rößler, O. Heczko, J. Buschbeck, B. Holzapfel, L. Schultz, and S. Fähler, New J. Phys. 13, 053029 (2011).

[19] A. G. Khachaturyan, S. M. Shapiro, and S. Semenovskaya, Phys. Rev. B 43, 10832 (1991).
[20] M. Zeleny, A. Sozinov, L. Straka, T. Björkman, and R. M. Nieminen, Phys. Rev. B 89, 184103 (2014).

[21] A. Bartels, R. Cerna, C. Kistner, A. Thoma, F. Hudert, C. Janke, and T. Dekorsy, Rev. Sci. Instrum. 78, 035107 (2007).

[22] H. Schäfer, V. V. Kabanov, M. Beyer, K. Biljakovic, and J. Demsar, Phys. Rev. Lett. 105, 066402 (2010).

[23] E. Möhr-Vorobeva, S. L. Johnson, P. Beaud, U. Staub, R. De Souza, C. Milne, G. Ingold, J. Demsar, H. Schaefer, and A. Titov, Phys. Rev. Lett. 107, 036403 (2011).

[24] H. Schaefer, M. Koerber, A. Tomeljak, K. Biljakovic, H. Berger, and J. Demsar, Eur. Phys. J. Spec. Top. 222, 1005 (2013).

[25] H. Schaefer, V. V. Kabanov, and J. Demsar, Phys. Rev. B 89, 045106 (2014).

[26] See Supplemental Material at http://link.aps.org/ supplemental/10.1103/PhysRevLett.115.076402 for more information on the acoustic properties of the samples as well as further information on the measurement methods, which includes Refs. [27-29].

[27] P. Leicht, A. Laptev, M. Fonin, Y. Luo, and K. Samwer, New J. Phys. 13, 033021 (2011).

[28] A. Bartels, T. Dekorsy, H. Kurz, and K. Köhler, Phys. Rev. Lett. 82, 1044 (1999).

[29] C. Thomsen, H. T. Grahn, H. J. Maris, and J. Tauc, Phys. Rev. B 34, 4129 (1986).

[30] S. O. Mariager, A. Caviezel, P. Beaud, C. Quitmann, and G. Ingold, Appl. Phys. Lett. 100, 261911 (2012).

[31] V. A. Chernenko, C. Segui, E. Cesari, J. Pons, and V. V. Kokorin, Phys. Rev. B 57, 2659 (1998).

[32] T. Kanomata, Y. Kitsunai, K. Sano, Y. Furutani, H. Nishihara, R. Y. Umetsu, R. Kainuma, Y. Miura, and M. Shirai, Phys. Rev. B 80, 214402 (2009).

[33] T. Kanomata et al., Phys. Rev. B 85, 134421 (2012).

[34] C. M. Li, H. B. Luo, Q. M. Hu, R. Yang, B. Johansson, and L. Vitos, Phys. Rev. B 84, 024206 (2011).

[35] P. Entel et al., Eur. Phys. J. B 86, 65 (2013).

[36] M. A. Uijttewaal, T. Hickel, J. Neugebauer, M. E. Gruner, and P. Entel, Phys. Rev. Lett. 102, 035702 (2009).

[37] S. O. Mariager et al., Phys. Rev. B 90, 161103 (2014). 